\title{
rempons
}

\section{Análisis del proceso tecnopolítico y de conectividad para el activismo feminista en Facebook, caso "Ni Una Menos Bolivia"}

\author{
Stephanie Morón Terrazas *
}

\section{Resumen}

Este artículo mostrará el proceso tecnopolítico y de conectividad que construyó el movimiento "Ni Una Menos Bolivia" en Facebook a partir del 2016, año en que se constituyó este colectivo para hacer frente mediante el activismo feminista a los casos de violencia de género y feminicidios ocurridos en este país. Se juntarán estas dos perspectivas teóricas ya que, un proceso tecnopolítico va de la mano de una cultura de conectividad (Van Dijck, 2013). La importancia de contar la experiencia del proceso tecnopolítico y de conectividad que construyó este movimiento en el caso boliviano es fundamental para entender el papel que juegan hoy en día los movimientos feministas en América Latina'.

\footnotetext{
* Licenciada en Ciencias Políticas por la Universidad Católica Boliviana "San Pablo". Maestra en Comunicación por la Universidad Iberoamericana de la Ciudad de México. Miembro del grupo de investigación sobre Generación, Subjetividad, Política y Activismo en el escenario posterior al movimiento \#YoSoy132 en México, del departamento de Comunicación de la Universidad Iberoamericana. Becaria doctoral en Comunicación por el Consejo Nacional de Ciencia y Tecnología CONACYT. Contacto: steph.m.terrazas@gmail.com
}

Morón Terrazas, Stephanie. "Análisis del proceso tecnopolítico y de conectividad para el activismo feminista en Facebook, caso 'Ni Una Menos Bolivia" en Zona Franca. Revista del Centro de estudios Interdisciplinario sobre las Mujeres, y de la Maestría poder y sociedad desde la problemática de Género, N²8, 2020 pp. 50-78. ISSN, 2545-6504 Recibido: 1 de agosto 2020; Aceptado: 12 de noviembre 2020

I Este artículo es parte de la investigación para la tesis de Maestría llamada: Análisis del proceso tecnopolítico y de conectividad en movimientos feministas dentro de Facebook. Caso: "Ni Una Menos Bolivia" realizada durante agosto 2017 - junio 2019 en México, también se contó con una estancia de investigación en la Facultad de Ciencias Políticas de la Universidad Católica Boliviana "San Pablo" gracias al financiamiento de la Beca de Movilidad CLACSO - CONACYT. Esta investigación fue presentada en su totalidad el día 10 de septiembre de 2019 para obtener el grado de Maestra en Comunicación por la Universidad Iberoamericana Ciudad de México, obteniendo mención honorífica.

Revista Zona Franca- Centro de estudios interdisciplinario sobre las mujeres (CEIM)- Maestría poder y sociedad desde la problemática de género (MG), Rosario, Argentina. ISSN, 2545-6504 http://zonafranca.unr.edu.ar/index.php/ZonaFranca| Número 28 (2020). 
Palabras clave: feminismo - tecnopolítica - conectividad - Ni Una Menos - Bolivia.

\section{Analysis of the technopolitical and connectivity processes for feminist activism on Facebook for "Ni Una Menos Bolivia"}

\section{Abstract}

This article will demonstrate the connectivity and technopolitical process that built the "Ni Una Menos Bolivia" movement on Facebook beginning in 2016, the year in which this collective was constituted with the express goal to help organize feminist activism responding to femicide and violence against women in the country. These two perspectives will be explored together due to the notion that a techno political process goes hand in hand with the culture of connectivity (Van Dijck, 2013). The fundamental importance of relating the experience of the technopolitical process and of the connectivity that built this movement in Bolivia is vital to further understand the roles that other feminist movements in Latin America exert in order to bring thousands of women together in response to the problem of gender-based violence.

Key words: feminism - technopolitics - connectivity - Ni Una Menos - Bolivia.

\section{Breve historia del feminismo en Bolivia y la participación política formal de las mujeres durante los siglos XX y XXI.}

Bolivia es un país que está situado en el centro de América del Sur. Según proyecciones a 2018 basadas en el último Censo de Población y Vivienda del año 2012, el Estado Plurinacional de Bolivia cuenta con aproximadamente 11.307.000 habitantes, de los cuales $49,6 \%$ son mujeres y $50,4 \%$ son hombres, además de un $62,2 \%$ de población indígena. Por área de residencia, $69,4 \%$ de la población vive en el área urbana y $30,6 \%$ en el área rural, según los últimos datos publicados por el Instituto Nacional de Estadística de Bolivia INE.

Estos indicadores son importantes para entender el contexto del movimiento feminista en Bolivia ya que, al ser un país donde el porcentaje femenino es casi

Revista Zona Franca- Centro de estudios interdisciplinario sobre las mujeres (CEIM)- Maestría poder y sociedad desde la problemática de género (MG), Rosario, Argentina. ISSN, 2545-6504 http://zonafranca.unr.edu.ar/index.php/ZonaFrancal Número 28 (2020). 
igual al porcentaje masculino y teniendo en cuenta el porcentaje de la población indígena, nos enfrentamos a un caso de estudio muy peculiar.

La historia del movimiento feminista en Bolivia, siempre estuvo marcado por la acción colectiva de mujeres procedentes de todas las clases sociales. Durante el Siglo XX, se produjo las primeras formas de participación política formal de las mujeres en asuntos de interés general y nacional, con una tendencia a que la militancia partidaria sea el vehículo de participación y de politización de las mujeres, con objetivos políticos nacionales de desarrollo a partir de sus posicionamientos ideológico políticos.

En la década de 1950, se dio un ascenso político de los sectores urbano populares y mineros con activa y creciente participación de mujeres. Esta inserción política no planteaba objetivos de igualdad de género, sino que su acción se vinculaba al proyecto político que sustentaban los partidos de la época.

La vuelta a la democracia en Bolivia durante la década de los ochenta inauguró un nuevo periodo en que las militantes mujeres empezaron a abrir nuevas rutas de alianzas dentro de diferentes partidos de izquierda, apostando por nuevas iniciativas bajo las banderas de la igualdad de género, de esta manera surgió una nueva forma de militancia, no orgánica, que permitió el avance del movimiento feminista.

El contexto de la década de los noventa fue esencial para empezar la participación política de manera "informal" de las mujeres en Bolivia a partir de la deconstrucción de significados culturales de aquella dominación patriarcal que una década antes, las mujeres feministas bolivianas habían empezado a tematizar y politizar. La aproximación a esta realidad, ahora contaba con el concepto de género, para explicar cómo se construyen en contextos históricos particulares, las relaciones sociales de dominación entre hombres y mujeres, haciendo evidente el desigual reparto del poder, y poniendo en la mesa de debate la violencia contra las mujeres en contextos de democracia, reivindicando la vigencia de los derechos de

Revista Zona Franca- Centro de estudios interdisciplinario sobre las mujeres (CEIM)- Maestría poder y sociedad desde la problemática de género (MG), Rosario, Argentina. ISSN, 2545-6504 http://zonafranca.unr.edu.ar/index.php/ZonaFranca| Número 28 (2020). 
las mujeres sobre sus cuerpos, fueron entre otros temas parte de la agenda de los movimientos feministas y ganancias de aquel periodo.

"La ampliación de la noción de poder centrado ya no en la relación con el Estado sino desplegándose en lo micro de lo cotidiano, en los intersticios de la sociedad, donde tienen lugar las asimetrías y jerarquías sociales entre hombres y mujeres, el desdoblamiento de lo político, "democracia en el país y en la casa", "lo personal es político" constituyeron un nuevo referente simbólico para interpelar las visiones hegemónicas sobre la democracia" (Zabala, 2010: 2).

En este contexto surge el colectivo "Mujeres Creando" que fue fundado el año 1992 por las activistas feministas: María Galindo, Mónica Mendoza y Julieta Paredes, en un barrio de la periferia de la ciudad de La Paz cuyo objetivo principal fue hacer un activismo feminista a través del arte y el graffiti bajo una propuesta de feminismo "no racista" que interpelaba a la élite de mujeres que vivían con privilegios y totalmente alejadas de la realidad de las mujeres indígenas cuyo trabajo no era valorado. Otro de sus objetivos fue la interpelación de los gobiernos bolivianos (históricamente de derecha hasta la llegada de Evo Morales en 2006) y de los partidos políticos.

Durante los primeros años del siglo XXI, se dio la emergencia de nuevos movimientos sociales dentro de la coyuntura política desde el 2006, "el paradigma de la diversidad cultural y el reconocimiento de un amplio catálogo de derechos bajo una orientación inclusiva, fortalece una presencia política masiva de mujeres, (Coordinadora de la Mujer, 2015: 16).

Bolivia al ser un Estado Plurinacional, teniendo una diversidad de culturas, de etnias y de clases sociales, hace que el movimiento feminista no está representado por un solo bloque, ni que se mueva bajo una idea única, por lo que hablamos de los feminismos. "El movimiento feminista y de mujeres en Bolivia enfrenta el reto de recrear sus prácticas y estrategias en un campo discursivo e identitario heterogéneo. Donde la noción de diversidades étnicas, culturales,

Revista Zona Franca- Centro de estudios interdisciplinario sobre las mujeres (CEIM)- Maestría poder y sociedad desde la problemática de género (MG), Rosario, Argentina. ISSN, 2545-6504 http://zonafranca.unr.edu.ar/index.php/ZonaFrancal Número 28 (2020). 
sociales, de género, sexuales, han fragmentado el viejo sujeto político feminista construido sobre la esencia unificadora de ser mujeres, que viene de compartir del mismo cuerpo, propio de la década de los 90s" (Zabala, 2010: 4).

Dentro de este contexto, surge el movimiento "Ni una Menos" abriendo un nuevo espacio para que las mujeres bolivianas pueden repensar el feminismo desde sus vidas cotidianas, concientizarse acerca de la violencia de género. Aunque el movimiento intenta incluir y exponer casos de mujeres en el área rural de Bolivia, la verdad es que es un colectivo fuertemente apoyado por mujeres de clase media de las principales ciudades de Bolivia: La Paz, Cochabamba y Santa Cruz, las cuales tienen acceso a internet móvil desde sus celulares, razón por la cual utilizan Facebook como su principal medio de comunicación en un país cuyo acceso a Internet es extremadamente caro.

\section{Contexto actual de la conectividad en Bolivia y uso de las redes sociales}

Bolivia se caracteriza por tener el Internet más lento y más caro de Sudamérica. La forma más común de conexión se da a través del Internet móvil ya que su acceso es primario y el Internet fijo es secundario.

En Bolivia existen tres empresas que dan servicio de Internet: ENTEL que es propiedad del Estado, VIVA Y TIGO que son empresas particulares. El consumo diario mínimo que ofrecen estas compañías es de 1000 megas que cuestan entre 20 a 30 Bs (Pesos Bolivianos) y equivalen entre 3 y 5 USD. Es decir que en un mes se gasta alrededor de 600bs a 900bs, entre 95 y 140 USD. Si un cliente quiere mayor cantidad de megas debe acceder a un plan semanal o mensual que duplican o triplican en costo a las tarifas mencionadas."

\footnotetext{
" Datos del artículo: El costo de navegar por Internet en Bolivia, publicado por el movimiento "Más y mejor Internet para Bolivia http://masymejorinternet.org.bo/sin-categoria/el-costo-de-navegar-porinternet-en-bolivia/_Consultado en 21 de abril de 2019.
}

Revista Zona Franca- Centro de estudios interdisciplinario sobre las mujeres (CEIM)- Maestría poder y sociedad desde la problemática de género (MG), Rosario, Argentina. ISSN, 2545-6504 http://zonafranca.unr.edu.ar/index.php/ZonaFranca| Número 28 (2020). 
Análisis del proceso tecnopolítico y de conectividad para el activismo feminista en Facebook, caso "Ni Una Menos Bolivia"

Según los resultados finales de la Encuesta Nacional de Opinión sobre Tecnologías de Información y Comunicación TIC realizada por la AGETIC en diciembre de 2017, de los 11. 307.000 de habitantes en Bolivia, el 78\% (8.82 millones) son internautas y $62 \%$ (7 millones) son usuarios activos de las redes sociales. Además, que un 59\% (6.70 millones) navegan desde un celular. La red social más usada en Bolivia es Facebook con 94\% de la población internauta. Presenta un total de 6.9 millones de usuarios activos en Bolivia, donde el $46 \%$ son mujeres y $54 \%$ son varones."II

Las actividades más frecuentes que los usuarios bolivianos realizan dentro de las redes sociales son: conectarse con amigos y familiares que representa un $97 \%$, ver fotos y videos con un $52 \%$ e informarse con $50 \%$. Mientras que la participación en debates y el apoyo a causas sociales están entre el $4 \%$ y $3 \%$, siendo las actividades menos realizadas. Las causas que más apoyan los bolivianos en las redes sociales son los derechos de los animales con un $60 \%$, luego están los derechos humanos con un $37 \%$ y en tercer lugar se encuentran los derechos de la mujer con un $28 \% .^{\text {IV }}$

Teniendo en cuenta el elevado costo de conexión a Internet en Bolivia, y sabiendo cuáles son los usos prioritarios de los usuarios, además de las causas que apoyan en redes sociales como Facebook y Twitter, estudiar el proceso de conectividad y tecnopolítico que construye \#NiUnaMenosBolivia en Facebook, es sumamente valioso e importante, siendo un caso único en la región con este contexto social, económico y tecnológico.

\section{Ni Una Menos Bolivia}

III Datos consultados del artículo Las redes sociales más utilizadas en Bolivia 2019, publicado por la página de marketing digital: MARRAQUETA http://www.marraquetamkt.com/redes-sociales/lasredes-sociales-mas-utilizadas-en-bolivia/ Consultado en: 20 de mayo de 2019.

IV Datos obtenidos de la Encuesta Nacional de Opinión sobre Tecnologías de Información y Comunicación TIC \#EncuestaTicBo https://agetic.gob.bo/pdf/ResultadosFinalesEncuestaTIC.pdf Consultado en: 20 de mayo de 2019.

Revista Zona Franca- Centro de estudios interdisciplinario sobre las mujeres (CEIM)- Maestría poder y sociedad desde la problemática de género (MG), Rosario, Argentina. ISSN, 2545-6504 http://zonafranca.unr.edu.ar/index.php/ZonaFrancal Número 28 (2020).

Página 55 
El objeto de estudio fue el grupo de Facebook del movimiento "Ni una Menos Bolivia" a partir de dos categorías: la primera es el proceso de conectividad para entender cómo funciona este grupo dentro de la plataforma de Facebook y cómo los usuarios se apropian de esta red social digital para publicar mensajes que exponen las demandas del movimiento "Ni una Menos Bolivia" con el fin de visibilizar el tema de violencia de género y feminicidios en este país sudamericano.

Es importante analizar el proceso de conexión entre los usuarios donde la "socialidad" y la tecnología se mezclan para dar lugar a plataformas que no son neutrales, por lo que es importante entender el medio donde se expresan opiniones, surgen debates y una serie de discursos sobre temas políticos, sociales, de género, de religión, entre otros a través del proceso de conectividad (Van Dijck, 2013).

En segundo lugar, se encuentra el proceso tecnopolítico que permite la apropiación tecnológica de una plataforma como Facebook para que los intereses y demandas políticas de un movimiento como fue el de los Indignados o $15 \mathrm{M}$ en España, se organice mediante redes sociales digitales.

Así se podrá observar cómo las mujeres activistas en Bolivia están utilizando distintas plataformas para traspasar los feminismos a un nuevo escenario tecnológico mediante el uso de la conectividad dentro de estas redes sociales digitales para que el debate sobre este tema y el discurso que se genera, pueda llegar a más personas y sea posible una organización masiva que permita llevar a cabo acciones concretas como marchas, huelgas y diversas actividades a favor de esta causa desde lo on-line hacia lo off-line y viceversa.

\subsection{Orígenes del movimiento "Ni una Menos" y características del}

\section{caso boliviano}

Revista Zona Franca- Centro de estudios interdisciplinario sobre las mujeres (CEIM)- Maestría poder y sociedad desde la problemática de género (MG), Rosario, Argentina. ISSN, 2545-6504 http://zonafranca.unr.edu.ar/index.php/ZonaFranca| Número 28 (2020).

Página 56 
Escogí el estudio de caso del movimiento "Ni una Menos Bolivia" que inspirado en los logros de su par "Ni Una Menos" en Argentina, salió a la luz por primera vez el 25 de noviembre de 2016 con una marcha multitudinaria en sus principales ciudades, su organización en redes sociales digitales empezó tres meses antes de la primera marcha en el mismo año y desde esa fecha es uno de los movimientos más grandes e importantes en Bolivia que se enmarca dentro de la lucha feminista a nivel global y que invita al activismo en contra de la violencia de género y feminicidios.

\section{Objetivos}

- Analizar el proceso de conectividad que se crea entre los miembros del grupo \#NiUnaMenosBolivia en Facebook usado tres constructos tecnoculturales: tecnología, usuarios y contenido.

- Analizar cómo se desarrolló el proceso tecnopolítico del movimiento feminista \#NiUnaMenosBolivia dentro del grupo de Facebook.

- Especificar los temas más importantes que son debatidos dentro del grupo de Facebook \#NiUnaMenosBolivia.

\section{Marco Teórico}

\subsection{Cultura de la Conectividad}

\footnotetext{
$\checkmark$ Nombre y consigna del movimiento feminista que retumbó en las redes sociales y que unió a toda América Latina en contra de la violencia de género. Empezó su actividad en redes sociales en junio de 2015 cuando un grupo de artistas y activistas en Argentina denunciaron el caso del asesinato de la adolescente Chiara Páez, replicándose muy pronto en varios países como Brasil, Perú, Chile y Bolivia.
}

Revista Zona Franca- Centro de estudios interdisciplinario sobre las mujeres (CEIM)- Maestría poder y sociedad desde la problemática de género (MG), Rosario, Argentina. ISSN, 2545-6504 http://zonafranca.unr.edu.ar/index.php/ZonaFranca| Número 28 (2020). 
La primera categoría analítica cuya teoría es desarrollada en el libro Cultura de la Conectividad, una historia crítica de las redes sociales por José Van Dijck el año 2013, está fundamentada en la teoría del actor - red y la economía política.

"Ofrecen valiosas ideas acerca de la transformación de la tecnología y la sociedad, y su combinación da por resultado el diseño de un modelo heurístico. Esto comprende dos configuraciones. La primera ayuda a 'desmontar los microsistemas' (...) aquella que considera a las plataformas (redes sociales) como constructos tecnoculturales y otra que las entiende en tanto estructuras socioeconómicas organizadas" (Van Dijck, 2013: 49).

A pesar de que la teoría del actor - red y la economía política desarrollan formas importantes de entender las complejidades de las redes sociales, Van Dijck las complementa para contribuir con un modelo de múltiples capas que se concentra en la disección de las plataformas individuales (microsistemas) entendidas como constructos tecnoculturales, pero también como estructuras socioeconómicas.

Se usará esta teoría porque nos permitirá entender las características que presenta la red social (en este caso Facebook) a través de la modalidad de grupo, para entender cómo se forma la "socialidad online" a través de constructos tecnoculturales (tecnología, usuarios y contenido) para formar un activismo feminista. La idea principal de la conectividad es la relación que hace entre lo "social" abarcando la conexión humana que se mezcla con la conectividad automática de las plataformas digitales.

\subsection{Tecnopolítica}

La segunda categoría analítica es la "Tecnopolítica" desarrollada por Javier Toret el año 2013 para explicar el caso del \#15M o "Movimiento de los Indignados" en España, está fundamentada en los conceptos sobre "el sistema red", "multitud conectada" y "ciberactivismo".

Revista Zona Franca- Centro de estudios interdisciplinario sobre las mujeres (CEIM)- Maestría poder y sociedad desde la problemática de género (MG), Rosario, Argentina. ISSN, 2545-6504 http://zonafranca.unr.edu.ar/index.php/ZonaFrancal Número 28 (2020).

Página 58 
El autor propone que las multitudes conectadas, surgen de una explosión emocional que deriva en un contagio estructurado por la tecnología propia de las redes sociales digitales a través de la conectividad de miles de usuarios que usan estas plataformas para comunicar mensajes concretos, propiciando conversaciones y debates sobre el problema social que los llama al activismo y que a la vez los empodera para salir a las calles a través de una organización previa dentro de la web.

Estas multitudes emergen con ciertos acontecimientos "aumentados", que se vuelven visibles gracias a la viralización de los mensajes que circulan por las redes sociales digitales como Facebook y Twitter. Se habla de hechos de aumentados para hacer referencia al juego performativo entre las capas físicas y digitales de un suceso que se extiende gracias a la posibilidad de: vivirlo, pre vivirlo y post - vivirlo tanto en las redes como en las calles, es así que la tecnopolítica va más allá del ciberactivismo. "Por ello, proponemos la noción de tecnopolítica como uso táctico y estratégico de las herramientas digitales para la organización, comunicación y acción colectiva como concepto clave para entenderlas" (Toret, 2013: 20).

Desde la perspectiva del sistema - red, la tecnopolítica se construye como la capacidad de estas multitudes conectadas para crear, organizar y llevar a cabo una acción colectiva que inventa formas de acción que se pueden dar en la red pero que no acaban en ellas.

\section{Metodología}

La perspectiva metodológica fue de carácter cualitativo, dentro del paradigma hermenéutico interpretativo puesto que esta investigación buscó un conocimiento consensuado a través de la interpretación del proceso tecnopolítico y de conectividad que crea el movimiento \#NiUnaMenosBolivia". La estrategia metodológica estuvo basada en las formas de interacción social, los mensajes y

Revista Zona Franca- Centro de estudios interdisciplinario sobre las mujeres (CEIM)- Maestría poder y sociedad desde la problemática de género (MG), Rosario, Argentina. ISSN, 2545-6504 http://zonafranca.unr.edu.ar/index.php/ZonaFrancal Número 28 (2020).

Página 59 
los discursos que se desarrollan dentro del grupo de Facebook "Ni una Menos Bolivia", por lo que fue fundamental la recolección de datos para generar explicaciones en relación al marco teórico planteado.

El punto de vista epistemológico fue inductivo, lo que permitió dar explicaciones a partir de los datos recabados en campo, sobre los que posteriormente se encuentran principios teóricos. Desde la lógica formal, el procedimiento inductivo es aquel que parte de lo particular hacia lo general; es la acción y efecto de extraer a partir de determinadas observaciones o experiencias particulares, el principio específico de cada una de éstas (Orozco, 2016: 99).

Teniendo en cuenta el reto que implicó construir una metodología idónea para analizar un fenómeno de comunicación dentro de las redes sociales digitales, específicamente dentro de Facebook, se recurrió al uso de la "Etnografía Virtual" desarrollada por Christine Hine ${ }^{\mathrm{VI}}$ como técnica de investigación cualitativa. Esta autora plantea la idea de "crisis de la etnografía" para explicar que esta ciencia ha cambiado con los años, y que ahora una variedad de campos disciplinarios como la sociología, la comunicación y la tecnología la ha adoptado para observar fenómenos más cotidianos en contraposición de la comprensión de culturas en lugares distantes como lo hace la tradición antropológica.

\begin{abstract}
"Tomaremos la etnografía como una práctica textual y una destreza adquirida y como una experiencia artesanal que pone en entredicho los fundamentos tradicionales basados en la presencia prolongada del investigador en un espacio físico determinado. El propósito de esta reevaluación de la etnografía es encontrar una nueva manera de lidiar
\end{abstract}

\footnotetext{
VI Socióloga de la ciencia y la tecnología. Profesora del departamento de Sociología de la Universidad de Surrey en Reino Unido. Tiene un enfoque particular en el papel que juegan las nuevas tecnologías en el proceso de construcción del conocimiento, además de un gran interés en el desarrollo de la etnografía en entornos técnicos y en "métodos virtuales" (el uso de Internet en la investigación social). En particular, ha desarrollado enfoques móviles y conectivos a la etnografía que combinan contextos sociales en línea y fuera de línea.
}

Revista Zona Franca- Centro de estudios interdisciplinario sobre las mujeres (CEIM)- Maestría poder y sociedad desde la problemática de género (MG), Rosario, Argentina. ISSN, 2545-6504 http://zonafranca.unr.edu.ar/index.php/ZonaFrancal Número 28 (2020). 
con algunos problemas que arrastraría esta perspectiva con respecto a Internet" (Hine, 2004: 57).

\subsection{Aplicación de la etnografía virtual para esta investigación}

Etnográficamente, se recopilaron las acciones que se desarrollaron dentro del funcionamiento del grupo en Facebook. Esto permitió analizar la conectividad que se ejerce entre los usuarios y el proceso tecnopolítico.

El análisis de datos se hizo mediante un proceso permanente de observación que fue ilustrado con capturas de imagen de los mensajes publicados en el grupo de Facebook del movimiento durante los meses de septiembre, octubre, noviembre y diciembre de 2016.

Se escogieron estas fechas para observar las publicaciones sobre la convocatoria a la marcha que se organizó el día 25 de noviembre en el centro de la ciudad de La Paz, para demostrar cómo se pasó de un activismo online hacia formas de protestas offline que tomaron las calles.

\section{Organización y análisis de la información}

\section{1 \#NiUnaMenos un movimiento de Twitter, es Ilevado a Facebook en el caso boliviano}

El movimiento "Ni Una Menos" nació en Argentina, siendo el primer país en la región que convocó movilizaciones a partir de junio de 2015 utilizando el nombre, la consigna y el hashtag feminista: \#NiUnaMenos en la plataforma Twitter.

La utilización de hashtags o etiquetas dentro de las redes sociales especialmente en Twitter, agrupa todos los mensajes sobre un tema en específico, permitiendo el desarrollo de un discurso cívico, político, social, económico, y de un sinfín de temas que dan como resultado un impacto comunicacional concreto.

Revista Zona Franca- Centro de estudios interdisciplinario sobre las mujeres (CEIM)- Maestría poder y sociedad desde la problemática de género (MG), Rosario, Argentina. ISSN, 2545-6504 http://zonafranca.unr.edu.ar/index.php/ZonaFranca| Número 28 (2020). 
"Twitter tiene cinco usos estratégicos: "como reacción a una política pública, como medio de expresión colectiva de un descontento, como medio de publicación de información ausente de la discusión pública y/o de los medios de comunicación tradicionales, como espacio de movilización ciudadana para impulsar una agenda y como instrumento de agregación de preferencias en torno a una política pública" (Merino, 2001:18).

En este sentido, el movimiento "Ni Una Menos Bolivia" siguiendo las formas de comunicación digital que desarrolló su par argentino, contó con una cuenta de Twitter Ilamada: @NiUnaMenosBol, además del grupo de Facebook \#NiUnaMenosBolivia.

Ambas cuentas fueron creadas en octubre de 2016, sin embargo, ocurrió una migración del debate de Twitter a Facebook, característica propia del caso boliviano, contrario a lo que pasó en el caso argentino, siendo Twitter la plataforma más usada para organizar y comunicar las acciones de este movimiento.

La cuenta de Twitter @NiUnaMenos_que es la oficial del movimiento argentino, contaba hasta mayo de 2019 con 69,5 mil seguidores.

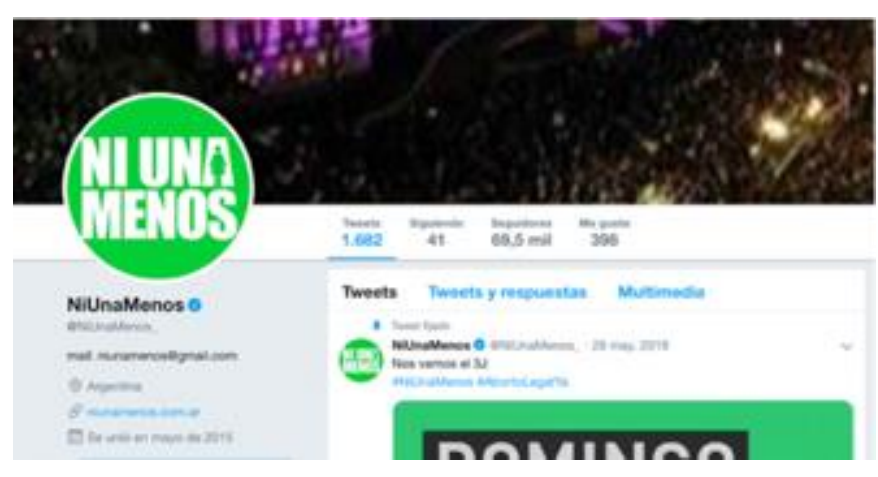

Imagen 1: cuenta de Twitter de Ni Una Menos Argentina

\section{https://twitter.com/NiUnaMenos}

Por otra parte, aunque la cuenta de Twitter @NiUnaMenosBol aún continúa activa, no existe una actualización desde el 7 de marzo de 2017, contando sólo 
con 893 seguidores hasta mayo de 2019, razón fundamental para que el debate y la acción de este movimiento se concentre en su grupo de Facebook, es así que, para esta investigación, se descartó el análisis de esta cuenta de Twitter.

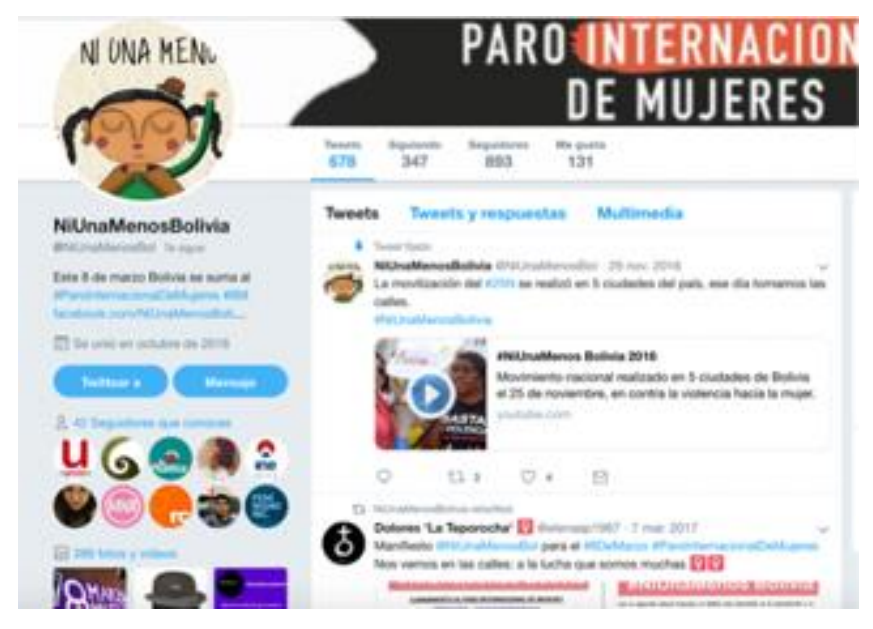

Imagen 2: cuenta de Twitter de Ni Una Menos Bolivia

https://twitter.com/NiUnaMenosBol

Este acontecimiento digital fue llevado a Facebook dentro del contexto boliviano porque es la red social digital más utilizada en Bolivia. En este sentido las usuarias bolivianas, aprovechan al máximo sus posibilidades de conexión para acumular mayor capital social, es así como la conectividad dentro de esta plataforma se desarrolla mediante la interconexión de las redes humanas por parte de los usuarios mezclada con la conectividad automatizada que genera Facebook mediante sus interfaces, promoviendo esta interconexión como un valor social.

Este valor social resulta clave para la participación política y cívica en el caso de los nuevos movimientos sociales que se generan dentro de estas plataformas, debido a la falta de espacios de debate y de medios de comunicación para expresar sentimientos de indignación, las redes sociales digitales como Facebook y Twitter se convierten en el espacio donde se generan nuevos procesos comunicativos para llevar a cabo revueltas y movilizaciones sociales.

Revista Zona Franca- Centro de estudios interdisciplinario sobre las mujeres (CEIM)- Maestría poder y sociedad desde la problemática de género (MG), Rosario, Argentina. ISSN, 2545-6504 http://zonafranca.unr.edu.ar/index.php/ZonaFranca| Número 28 (2020). 


\subsection{La Cultura de la Conectividad dentro de un grupo de Facebook,}

caso: "Ni Una Menos Bolivia"

Dentro de la plataforma Facebook, encontramos a los grupos, que son espacios creados para agrupar a una comunidad de usuarios que además de contar con sus cuentas personales, deciden crear o unirse a un grupo para compartir intereses, conocimiento e información con cualquier persona en un ámbito mucho más grande del que ofrece un perfil personal. Los grupos de Facebook se clasifican en función de la temática y la privacidad, existiendo tres tipos de grupos en Facebook:

- Abiertos: Es público, toda la información está a la vista y no es necesario hacer una solicitud de acceso.

- Cerrados: Hay que hacer una solicitud para poder unirse al grupo y es el administrador/es del grupo quien aprueba tu solicitud.

- Secretos: Un grupo que se crea por una persona, no es visible, y la única forma de entrar es que el creador de este grupo de Facebook te mande invitación.

En el caso del grupo nos encontramos con un grupo cerrado es así que el primer resultado del objeto de estudio arroja la característica de tener una conectividad limitada.

Revista Zona Franca- Centro de estudios interdisciplinario sobre las mujeres (CEIM)- Maestría poder y sociedad desde la problemática de género (MG), Rosario, Argentina. ISSN, 2545-6504 http://zonafranca.unr.edu.ar/index.php/ZonaFrancal Número 28 (2020). 


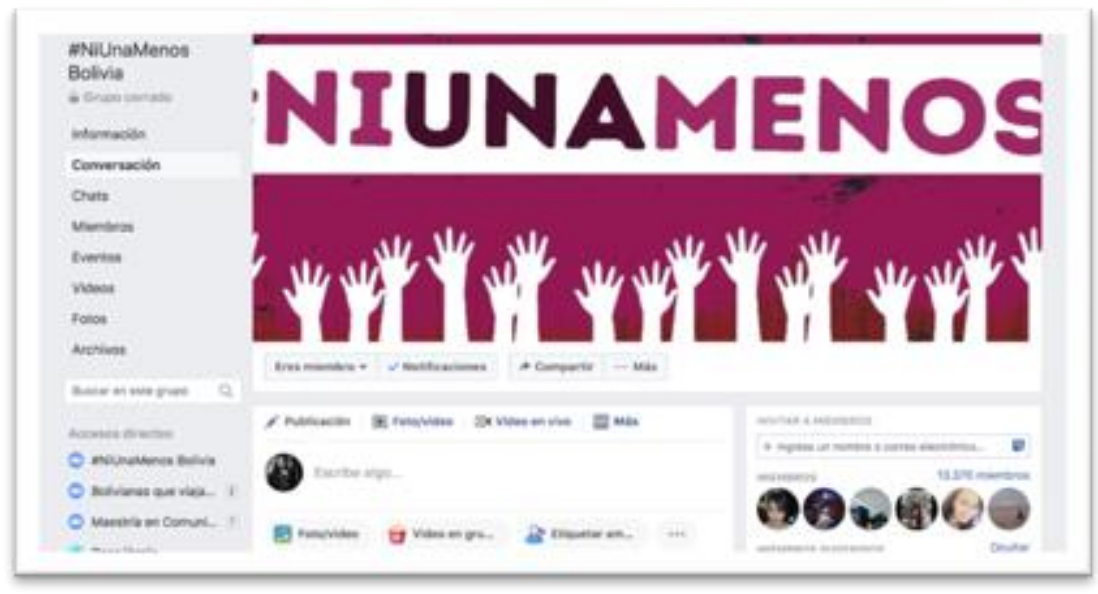

Imagen 4: Portada del grupo \#NiUnaMenos Bolivia en Facebook

\section{https://www.facebook.com/groups/323210768016095}

Otra característica importante de este grupo es que, a pesar de ser cerrado, cuenta con un total de 13,372 miembros ${ }^{\mathrm{VII}}$, cifra bastante elevada. La razón por la que esta cuenta es cerrada es porque en un inicio, la convocatoria con la que inició el movimiento fue a través de un llamado de carácter ciudadano, por lo que las activistas invitaron a todo aquel que quisiera unirse y luchar en contra de la violencia de género y feminicidios en Bolivia.

A través de la observación que permitió la etnografía virtual, es evidente que, en el transcurso de los meses, viendo la polémica que se generaba en los comentarios de las publicaciones, tomaron la decisión de convertir al grupo en un espacio cerrado.

Bajo esta perspectiva, desde la teoría nos encontramos con un caso particular dentro de la conectividad y la socialidad en red, como la define José Van Dijck (2013), pues al ser un grupo cerrado y manejado a criterio de las administradoras, la subjetividad para elegir quien es admitido o no, interfiere con la VII Último dato actualizado del grupo, consultado en: 09 de mayo de 2019 https://www.facebook.com/groups/323210768016095/

Revista Zona Franca- Centro de estudios interdisciplinario sobre las mujeres (CEIM)- Maestría poder y sociedad desde la problemática de género (MG), Rosario, Argentina. ISSN, 2545-6504 http://zonafranca.unr.edu.ar/index.php/ZonaFranca| Número 28 (2020). 
facilitación y la potencialidad de la interconexión como valor social, sin embargo, en la realidad podemos observar que, a pesar de ser un grupo cerrado, al contar con un más de 13 mil miembros, la potencialidad de interconexión aún sigue siendo muy alta, y el valor social sigue presente al momento de poner los filtros de admisión, como forma de asegurar que esta plataforma será un espacio de debate formal.

\subsection{Proceso Tecnopolítico del Movimiento "Ni una Menos Bolivia"}

Entenderemos al proceso tecnopolítico como: la capacidad de las multitudes conectadas (Toret, 2015; Rovira 2017) para crear, organizar y llevar a cabo una acción colectiva que inventa formas de acción que se pueden dar en la red pero que no acaban en ellas.

De esta manera, a través de la técnica de Etnografía Virtual, se explicará el proceso tecnopolítico que ha construido el movimiento "Ni Una Menos Bolivia" a través de hechos específicos que demuestra la creación, y la organización dentro de esta plataforma para llamar a la acción colectiva online y offline.

\subsubsection{Organización de asambleas y posicionamiento político del movimiento dentro del grupo de Facebook}

El movimiento "Ni Una Menos Bolivia" creó el grupo de Facebook el 20 de agosto de 2016, dos semanas después comenzaron la acción colectiva mediante la publicación de los acuerdos de la primera asamblea que tuvo lugar el 3 de septiembre de 2016. El documento que sustenta los primeros acuerdos a los que se llegó fue subido el 5 de septiembre del mismo año con el nombre "Apthapivill de ideas".

VIII El Apthapi se refiere a la comida que se comparte entre miembros de una comunidad o familia, propia de la cultura Aymara donde cada persona lleva algo que cultivó para compartir. En el contexto de la asamblea se refiere a una "lluvia de ideas".

Revista Zona Franca- Centro de estudios interdisciplinario sobre las mujeres (CEIM)- Maestría poder y sociedad desde la problemática de género (MG), Rosario, Argentina. ISSN, 2545-6504 http://zonafranca.unr.edu.ar/index.php/ZonaFrancal Número 28 (2020). 
Esta publicación tiene un acceso a un documento en Word subido por una de las administradoras. El primer acuerdo señala la organización de una “movilización nacional ciudadana y popular, no institucional” para el día 25 de noviembre de 2016 en la ciudad de La Paz.

Esto nos da a entender de que la forma de organización del movimiento no nació dentro de Facebook ni de Twitter, sino que ocurrieron asambleas organizadas previamente, por un grupo de mujeres activistas feministas que decidieron replicar la experiencia de Argentina en Bolivia. Para ello, la asamblea ocurrió en el espacio offline, y se usó el grupo de Facebook para comunicar el mensaje de manera masiva, invitando a los miembros del grupo a realizar publicaciones serias con fuentes confiables, así como a unirse a las distintas comisiones que se propusieron para dar sustento al movimiento.

Por otra parte, se manifiesta el deseo de organizar más movilizaciones en el interior de Bolivia, como en la ciudad de Santa Cruz, y se hace énfasis que el tipo de publicaciones que serán aceptadas en el grupo deben ser útiles para visibilizar el problema de violencia de género y feminicidios, usando cifras y documentos con validez académica, así como evitar hacer comentarios discriminadores o que vayan en contra de la libertad político- partidaria de cada miembro del grupo.

Revista Zona Franca- Centro de estudios interdisciplinario sobre las mujeres (CEIM)- Maestría poder y sociedad desde la problemática de género (MG), Rosario, Argentina. ISSN, 2545-6504 http://zonafranca.unr.edu.ar/index.php/ZonaFrancal Número 28 (2020). 

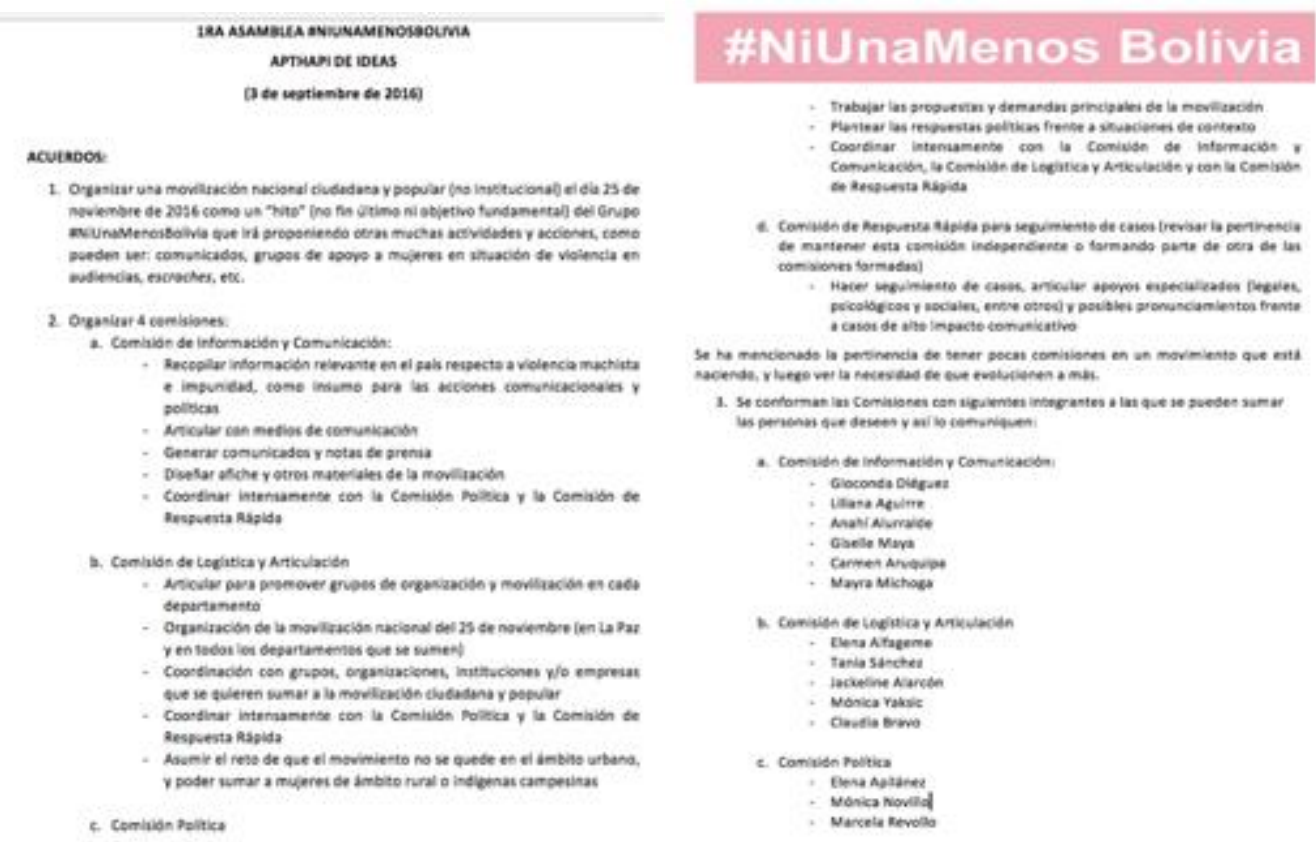

Revista Zona Franca- Centro de estudios interdisciplinario sobre las mujeres (CEIM)- Maestría poder y sociedad desde la problemática de género (MG), Rosario, Argentina. ISSN, 2545-6504 http://zonafranca.unr.edu.ar/index.php/ZonaFrancal Número 28 (2020). 
Análisis del proceso tecnopolítico y de conectividad para el activismo feminista en Facebook, caso "Ni Una Menos Bolivia"

\section{\#NiUnaMenos Bolivia}

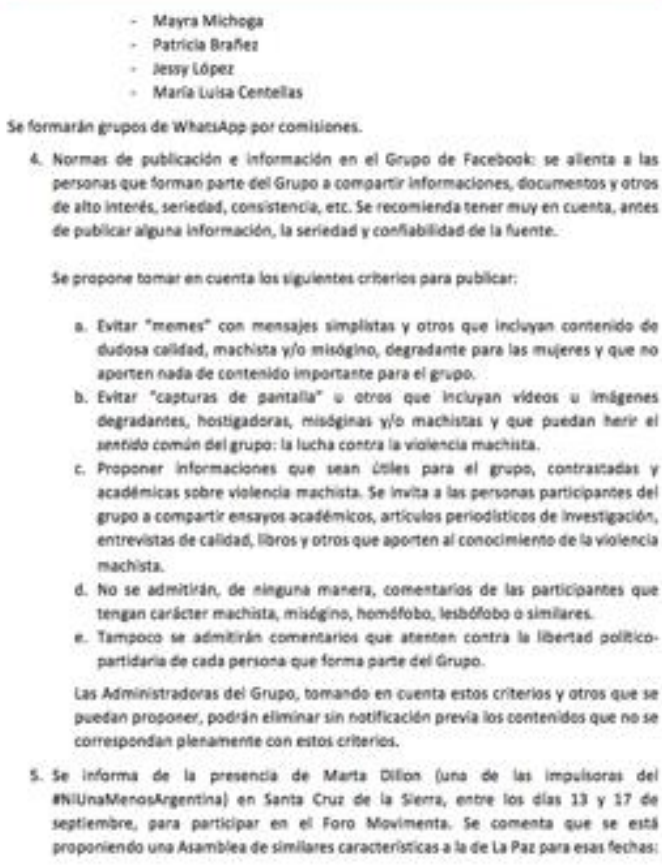

4. Normas de putlicacion e informaciton en el Gerpo de Facebook se alenta a las personas que Soman parte cel Grupo a comparti informaciones, focomestos y otros te aho interts. seriedid, consistencla, etc. Se reconienda sener muy en cuesta, ames

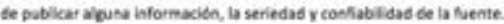

Se sropose somar en cuenta los icuientes criberies para publeat;

- Eviar "menes" con mensajes simplistas y otros que incloyn contenido on budos caldad, machisa yjo misogina, degradante para las muleres y que no aporten nada de contenibo inportaste para el cropo

b. Evitar "casturas de samtals" o etres que hclupan videor o imigtenes

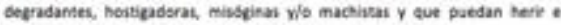
aentido comin bei erupo: la lucha costa la volencia muctits.

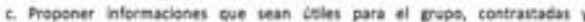

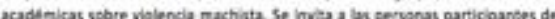

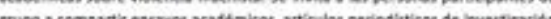

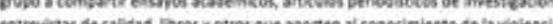

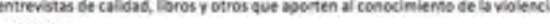
machista.

C. No se admitian, de sineuna masen, comentarios de las particantes que

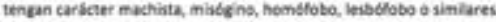

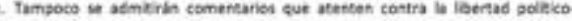
partidaria de cada periosa que forma garse cel Grugo.

Las Administradoras del Grupo, bomands en cuenta estos criterios y otros que se Dsedan prososer, podis eliminar in socificacien previa los cemtenides que no se correipsndan plenenteme can eitor criberies.

5. Se informa de la presentia de Mata Diton funa do in impultorat del

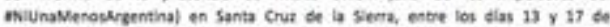
regtiembre, gars participar on ef Foro Movimenta. Se comenta que se etes

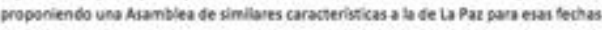

\section{\#NiUnaMenos Bolivia}

se convocará a las companeras de Santa Cruz de la Sierra en unot dias con la fecha, herario y lucer definide

6. Se anima a que se ample un poco el nimero de atminstradoras del grupo de Faceboek|

Imagen 5: Acuerdos de la 1ํAsamblea de \#NiUnaMenosBolivia publicada el 16 de septiembre de 2016 en el grupo de Facebook

https://www.facebook.com/groups/323210768016095

Los acuerdos que llegaron durante la primera reunión de comunicaciones realizada el jueves 15 de septiembre de 2016, dan muestra del proceso tecnopolítico que se suscitó en el desarrollo de las acciones que organizó el movimiento y que se comunicó a través de esta plataforma.

Revista Zona Franca- Centro de estudios interdisciplinario sobre las mujeres (CEIM)- Maestría poder y sociedad desde la problemática de género (MG), Rosario, Argentina. ISSN, 2545-6504 http://zonafranca.unr.edu.ar/index.php/ZonaFranca| Número 28 (2020). 
Una de los puntos más emblemáticos de esta reunión fueron los lemas que se crearon para esta movilización. El principal fue: "No nací mujer para morir por serlo", seguido de otros como: "vivas nos queremos"; "somos el grito de las que ya no tienen voz"; "el machismo mata"; "ni una mujer menos, ni una muerta más"; "cuando la sangre es de una mujer maltratada, la sangre es de todas"; "si tocan a una, respondemos todas"; "libres seremos"; "con ropa o sin ropa este cuerpo no se toca".

También se encuentra el Documento de Word con el posicionamiento político que creó el movimiento, dejando en claro que la movilización es resultado de la impunidad del sistema judicial boliviano, cuyo acceso es difícil, especialmente para las familias de las víctimas de feminicidios, a pesar de existir la Ley $348^{\mathrm{IX}}$ en Bolivia. Además, este movimiento responde a las acciones históricas del movimiento feminista y de mujeres que desde todos los rincones de Bolivia interpelan al Estado y a la sociedad para la construcción de políticas en contra de la violencia de género y la modificación de patrones culturales que propician un ambiente de discriminación y violencia en contra de la mujer.

De esta manera, en los primeros meses del movimiento, se puede encontrar las formas de organización que se implementaron mediante asambleas para la elaboración de acuerdos, y las formas comunicativas que usaron con el uso del grupo de Facebook para masificar el mensaje y sumar más personas a las movilizaciones.

IX Ley para garantizar a las mujeres una vida libre de violencia No 348 promulgada por el ex Presidente Evo Morales el 9 de marzo de 2013, siendo Bolivia el país con mayor número de feminicidios en Latinoamérica. Según la encuesta publicada en el Latinobarómetro de 2018 el promedio de violencia de género en la región es de $24 \%$, mientras que en Bolivia asciende al $34 \%$, lo que equivale a 10 puntos por encima del porcentaje regional. De esta forma, esta investigación pretende mostrar la realidad de este problema que se vive actualmente en Bolivia y cuáles son las razones fundamentales por las que se inició el movimiento "Ni Una Menos".

Revista Zona Franca- Centro de estudios interdisciplinario sobre las mujeres (CEIM)- Maestría poder y sociedad desde la problemática de género (MG), Rosario, Argentina. ISSN, 2545-6504 http://zonafranca.unr.edu.ar/index.php/ZonaFranca| Número 28 (2020). 


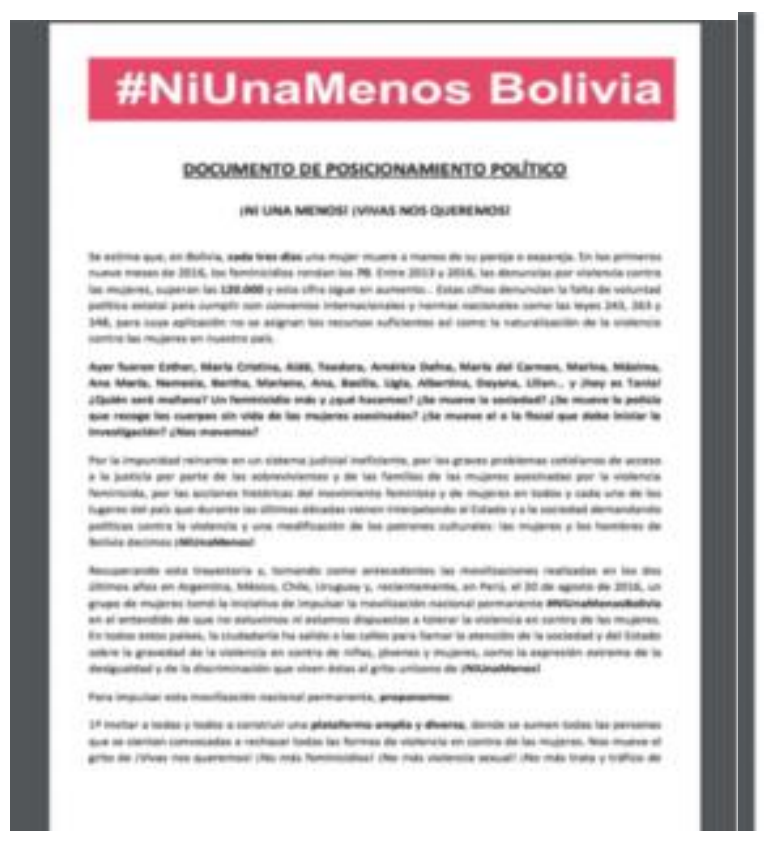

\section{\#NiUnaMenos Bolivia}

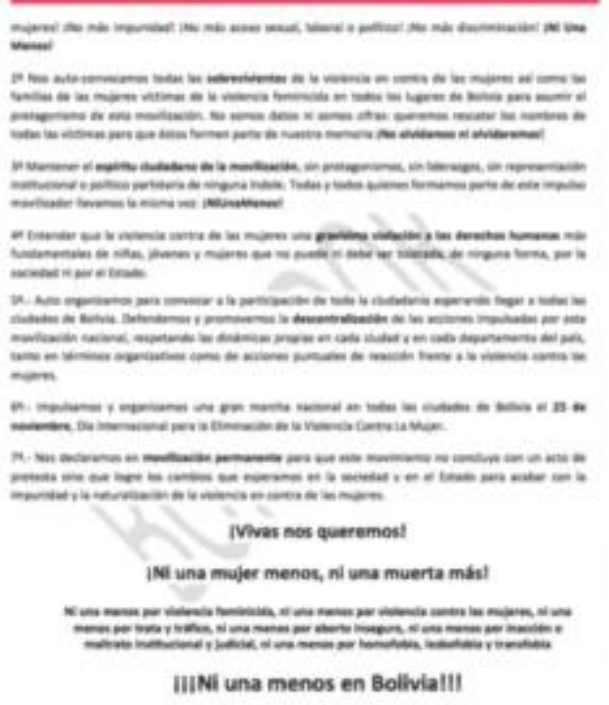

Imagen 6: Posicionamiento Político de \#NiUnaMenos Bolivia

De esta manera, el movimiento "Ni Una Menos Bolivia" propuso:

- La construcción de una plataforma amplia y diversa, donde se unan todas las personas que se sientan convocadas a rechazar todas las formas de violencia en contra de las mujeres.

- La auto-convocatoria de todas las sobrevivientes de la violencia de género, así como las familias de las víctimas de la violencia feminicida de todos los rincones de Bolivia.

- Mantener el espíritu ciudadano de la movilización, sin protagonismos, ni liderazgos, sin representación institucional o político partidaria.

Revista Zona Franca- Centro de estudios interdisciplinario sobre las mujeres (CEIM)- Maestría poder y sociedad desde la problemática de género (MG), Rosario, Argentina. ISSN, 2545-6504 http://zonafranca.unr.edu.ar/index.php/ZonaFranca| Número 28 (2020). 
- Entender que la violencia contra las mujeres es una gravísima violación a los Derechos Humanos que no debe ser tolerada por la sociedad ni por el Estado.

- Promover la descentralización de las acciones impulsadas por esta movilización nacional, respetando las dinámicas propias de cada ciudad y en cada departamento de Bolivia, tanto en términos organizativos como acciones concretas.

- Declaración de movilización permanente para que el movimiento no concluya con un acto de protesta, sino que logre los cambios que se espera en la sociedad y en el Estado, para acabar con la impunidad y la naturalización de la violencia en Bolivia.

Es así como se formó el proceso tecnopolítico del movimiento "Ni Una Menos Bolivia" entendido como el "uso táctico y estratégico de las herramientas digitales para la organización, comunicación y acción colectiva" (Toret, 2013: 20). De esta manera, observamos que las administradoras del grupo, utilizaron la plataforma del grupo de Facebook, para subir los documentos en Word que se realizaron durante las asambleas, tanto con formas tanto programáticas como prefigurativas de organización.

La forma programática hace referencia a las políticas de organización "formales" como lo son las asambleas, recurso utilizado por las mujeres que decidieron llevar la movilización nacional a nombre de \#NiUnaMenosBolivia para organizar el movimiento en comisiones y posteriormente crear un pliego de demandas y el posicionamiento político del movimiento.

La forma prefigurativa aparece cuando la acción colectiva creada en las asambleas va permeando en las redes, creando procesos comunicativos en el grupo de Facebook para propagar los mensajes de la posición ideológica, las formas de organización mediante comisiones y las demandas del movimiento para que todas las personas interesadas puedan sumarse a estas movilizaciones.

Revista Zona Franca- Centro de estudios interdisciplinario sobre las mujeres (CEIM)- Maestría poder y sociedad desde la problemática de género (MG), Rosario, Argentina. ISSN, 2545-6504 http://zonafranca.unr.edu.ar/index.php/ZonaFrancal Número 28 (2020). 


\section{Conclusiones}

Después de la observación realizada al grupo "Ni Una Menos Bolivia", usando la teoría de conectividad el análisis se enfocó en tres constructos tecnoculturales: tecnología, usuarios y contenido.

En el tema de tecnología se observó que los algoritmos creados a partir del procesamiento de los datos, dio lugar a una arquitectura de codificación propia del grupo \#NiUnaMenos Bolivia, filtrando la información de los perfiles personales de las administradoras y usuarias con mayor interacción, con el contenido expuesto en el grupo a partir de temas específicos sobre violencia de género y movilizaciones en defensa de los derechos de la mujer.

El papel que desarrollaron las usuarias dentro del grupo de Facebook se da desde una lógica participativa que las convierte en receptoras, consumidoras, productoras y partícipes de la cultura de conectividad, de esta manera, se entiende a las usuarias como prosumers, creando una socialidad online inmediata a través de la participación.

El contenido que se produce va de la mano con la participación de las usuarias, produciendo opiniones acerca de aquello les gusta, lo que desean o y lo que les interesa. Los gustos en común afianzan vínculos y descubren afinidades de grupo, creando valiosa información acerca de tendencias y preferencias de consumo. No obstante, como el objeto de estudio analizado responde a un grupo de Facebook formado con un fin estrictamente activista, los contenidos expuestos propician debates y conversaciones para expresar sentimientos de adhesión o de rechazo hacia las temáticas feministas y a las acciones del movimiento.

De esta manera, la cultura de conectividad creada dentro de este espacio, se dio mediante un proceso de cuantificación social creada mediante la relación: conexión humana/conectividad automatizada, manejadas por el software y el protocolo de Facebook, permitiendo procesos comunicativos dentro de una

Revista Zona Franca- Centro de estudios interdisciplinario sobre las mujeres (CEIM)- Maestría poder y sociedad desde la problemática de género (MG), Rosario, Argentina. ISSN, 2545-6504 http://zonafranca.unr.edu.ar/index.php/ZonaFranca| Número 28 (2020). 
comunidad muy grande que replica una y mil veces el imperativo de "compartir información" como lo señala José Van Dijck.

La contribución principal de las usuarias, radica en la formación de identidad a través de la participación activa y el compromiso feminista, generando una colectividad como sinónimo de conectividad automatizada, donde las usuarias se empoderan mediante el uso político que le dan a la plataforma.

Dentro de los principales hitos que se desarrollaron a partir del año 2016, que se consideraron como parte fundamental del proceso tecnopolítico del movimiento se centraron en convocatorias para asambleas mediante reuniones en espacios físicos donde se llegaron a una serie de acuerdos que posteriormente, fueron publicados en el grupo de Facebook, utilizando esta plataforma como una herramienta de comunicación clave para la expansión de los mensajes.

Por lo tanto, "Ni Una Menos Bolivia" no es un movimiento que nace desde Facebook, al contrario, parte de una organización tradicional y utiliza esta plataforma como medio potencializador de la acción colectiva, siendo un paso clave para el proceso tecnopolítico.

Dentro de los temas más relevantes que se debaten dentro del grupo se encuentran:

- Ideología del movimiento "Ni Una Menos" en Bolivia y a nivel global.

- Despenalización del aborto en Bolivia.

- Cifras de feminicidios en Bolivia.

- Casos específicos de violencia de género y feminicidios ocurridos en Bolivia.

- Crítica a la Ley 348.

- Recuento de las marchas y performances realizados por el $8 \mathrm{M}$ y $25 \mathrm{~N}$ con álbumes fotográficos.

Revista Zona Franca- Centro de estudios interdisciplinario sobre las mujeres (CEIM)- Maestría poder y sociedad desde la problemática de género (MG), Rosario, Argentina. ISSN, 2545-6504 http://zonafranca.unr.edu.ar/index.php/ZonaFrancal Número 28 (2020). 
Referente a los tipos de mensajes más usados dentro del grupo, se encuentran las publicaciones que sólo contienen texto, álbumes fotográficos, e ilustraciones con algún mensaje o cifras de casos de violencia de género y feminicidios. Otro tipo de contenido son las noticias que se publican, la fuente principal son periódicos bolivianos en su versión digital como LA RAZÓN, PÁGINA SIETE, EL DEBER y OPINIÓN que son publicados nuevamente dentro del grupo.

De esta manera se observó cómo el movimiento "NiUnaMenosBolivia" fue capaz de construir una cultura de conectividad y un proceso tecnopolítico propio para llevar a cabo una serie de movilizaciones a nivel nacional, y a poner en la mesa de debate el gran problema de la violencia de género y la ineficacia de la Ley 348.

Desde el punto de vista teórico, fue importante analizar el proceso de conectividad para entender cómo funciona la interacción social dentro de un grupo de Facebook con la finalidad de hacer un activismo feminista, mediante el funcionamiento de la plataforma a través de la tecnología, los datos y los usuarios, teniendo siempre presente que las redes sociales digitales no son neutras y que justamente dependen de estos constructos tecnoculturales para su desarrollo y consolidación.

Por otra parte, después de explorar la situación de conectividad en Bolivia, al ser el Internet más caro y lento de Sudamérica, el grupo de Facebook "Ni Una Menos Bolivia, con más de 13 mil usuarios, es una muestra del valor que tiene en participación e interacción, haciendo posible la visibilización de la violencia de género en Bolivia tanto en redes sociales como en las calles.

El aporte de esta investigación está centrado en resaltar el activismo a partir de la comunicación digital creado por el movimiento "Ni una Menos Bolivia" que es un referente feminista para las nuevas generaciones de mujeres bolivianas, y justamente por esa razón, el estudio de su proceso tecnopolítico y de conectividad

Revista Zona Franca- Centro de estudios interdisciplinario sobre las mujeres (CEIM)- Maestría poder y sociedad desde la problemática de género (MG), Rosario, Argentina. ISSN, 2545-6504 http://zonafranca.unr.edu.ar/index.php/ZonaFranca| Número 28 (2020). 
dentro de Facebook es un caso particular, ya que estos movimientos suelen tener mayor participación e interacción en Twitter.

Por esta razón, es importante seguir creando espacios y programas de prevención de violencia para que las mujeres y hombres bolivianos puedan formarse desde niños, permitiendo una deconstrucción del machismo fuertemente enraizado en la sociedad boliviana, y que a partir del feminismo y de la resistencia, permita una militancia que sea capaz de realizar un activismo diverso, usando esa performatividad propia de las luchas feministas.

\section{Bibliografía}

AVALOS, Juan Manuel. (2018). Activismos Políticos contemporáneos. Juventudes, Movilizaciones y Comunicación en Guadalajara. Instituto Mexicano de la Juventud.

Autoridad de Regulación y Fiscalización de Telecomunicaciones y Transportes ATT (2019). Estado de Situación del Internet en Bolivia. Recuperado de https://att.gob.bo/content/situaci\%C3\%B3n-del-internet-en-bolivia

BAER, Hester. (2016). "Redoing feminism: Digital activism, body politics, and neoliberalism". Feminist Media Studies, 16(1), 17-34. Recuperado de https://www.tandfonline.com/doi/full/10.1080/14680777.2015.1093070

BOIX, Montserrat. (2015). "Desde el ciberfeminismo hacia la tecnopolítica feminista". En Revista Pillku. Recuperado de http://pilku.org/article/desde-elciberfeminismo-hacia-la- tecnopolitica-fem/

BLAZQUEZ, Nora; FLORES, Fátima; RíOS, Maribel. (2010). Investigación Feminista. Epistemología, Metodología y Representaciones Sociales. Centro de Investigaciones Interdisciplinarias en Ciencias y Humanidades. Centro Regional de Investigaciones Multidisciplinarias. Universidad Nacional Autónoma de México.

CANCELO, Mercedes y GADEA, Gilda. (2013). Empoderamiento de las redes sociales en las crisis institucionales, (versión electrónica), España: Vivat Academia, año XV, $\mathrm{n}^{\circ} 124 . \quad$ Recuperada de http://www.vivatacademia.net/index.php/vivat/article/view/185

Revista Zona Franca- Centro de estudios interdisciplinario sobre las mujeres (CEIM)- Maestría poder y sociedad desde la problemática de género (MG), Rosario, Argentina. ISSN, 2545-6504 http://zonafranca.unr.edu.ar/index.php/ZonaFranca| Número 28 (2020). 
CASTELLS, Manuel. (2012). Redes de indignación y Esperanza. Alianza Editorial.

Coordinadora de la Mujer, Instituto Internacional para la Democracia y la Asistencia Electoral IDEA Internacional. (2015). Participación de las mujeres en el Estado. La Paz - Bolivia.

FUCHS, Christian. (2009). Information and communication technologies and society. A contribution to the critique of the political economy of the internet". European Journal of Communication. (pp. 69-87).

FUCHS, Christian. (2014). Social Media, a Critical Introduction. SAGE Publications Inc.

GAMBA, Susana. (2008). "Feminismo. Historias y Corrientes". Artículo publicado en: Red, El periódico feminista. Recuperado en: http://www.mujeresenred.net/spip.php?article1397

GUTIÉRREZ - RUBí, Antoni. (2014). TECNOPOLítICA. El uso y la concepción de nuevas herramientas tecnológicas para la comunicación, la organización y la acción política colectivas. España: Ediciones Grafiko.

HINE, Cristine. (2000). Etnografía Virtual. Cultura Libre: Estados Unidos.

HINE, Cristine. (2015). Ethnography for the Internet: Embedded, Embodied and Everyday. Bloomsbury Academc: London.

MORÓN, Stephanie. (2019). Análisis del proceso tecnopolítico y de conectividad en movimientos feministas dentro de Facebook. Caso: 'Ni Una Menos Bolivia. (Tesis de Maestría). Universidad Iberoamericana, Departamento de Comunicación , Ciudad de México. Recuperada en: http://ri.ibero.mx/handle/ibero/2428

ROVIRA, Guiomar. (2017). Activismo en red y multitudes conectadas. Universidad Autónoma Metropolitana - Unidad Xochimilco.

ROVIRA, Guiomar. (2018). "El devenir feminista de la acción colectiva: las redes digitales y la política de prefiguración de las multitudes conectadas". Enrevista Teknocultura 15(2), 223-240.

Revista Zona Franca- Centro de estudios interdisciplinario sobre las mujeres (CEIM)- Maestría poder y sociedad desde la problemática de género (MG), Rosario, Argentina. ISSN, 2545-6504 http://zonafranca.unr.edu.ar/index.php/ZonaFrancal Número 28 (2020). 
TORET, Javier. (2013). Tecnopolítica: la potencia de las mutitudes conectada. Internet Interdisciplinary Institute: España.

TERZIAN, Polly. (2017). "The Ni Una Menos Movement in 21st Century Argentina: Combating more than femicide. Dickinson College, Honor Theses. Paper 228.

VAN DIJCK, José. (2013). La cultura de la conectividad. Siglo Veintiuno Editores.

ZABALA, Lourdes. (2010). "Del Feminismo a los Feminismos en Bolivia". Coordinadora de la Mujer. Recuperado en: http://www.coordinadoradelamujer.org.bo/observatorio/archivos/publicaciones/artic ulo feminismos en bolivia lourdes zabala 58.pdf

Revista Zona Franca- Centro de estudios interdisciplinario sobre las mujeres (CEIM)- Maestría poder y sociedad desde la problemática de género (MG), Rosario, Argentina. ISSN, 2545-6504 http://zonafranca.unr.edu.ar/index.php/ZonaFrancal Número 28 (2020). 